\title{
A Composite Model for an Intelligent Device-To-Device Communication in the Internet of Things
}

\author{
B. Kalpana, R. Sanjay
}

\begin{abstract}
Similar to how humans communicate, devices will have to communicate with each other in the Internet of Things (IoT) ecosystem. Where the devices themselves become the users of the knowledge or the data that is acquired. Hence we can say that device-to-device communication becomes an intrinsic part of IoT. Devices have to communicate autonomously with each other without any centralized monitoring system. It will be more powerful when the devices can communicate in a multi-hop manner rather than a single-hop manner where the information when and where needed can be accumulated and used. This leads to a need where there must be a group communication between the devices in a particular network. Hence information about the network and the environment in which the devices operate can be identified and the relevant knowledge can be transferred to a peer network or a device for further information gathering or the peer nodes can be used as intermediate nodes in the multi-hop network. Here we are proposing a network of multi-hop interconnected intelligent IoT devices that can communicate among its peer devices gather information and send the information in a multi-hop fashion making using of the adjacent networks or clusters.
\end{abstract}

Keywords: Internet of Things (IoT), Device to Device communication, multi hop, Centralized monitoring system.

\section{INTRODUCTION}

$\mathrm{W}_{\mathrm{i}}$ increasingly interconnected at an unprecedented scale. In modern times the Internet of Things (IoT) is emerging as the latest trend for communication and device control. The Internet of Things (IoT) represents a diverse technology and usage with unprecedented business opportunities and risks. The Internet of Things is changing the dynamics of the security industry \& reshaping it. It allows data to be transferred seamlessly among physical devices to the Internet. Internet of Things (loT) is a global infrastructure for the information society, enabling advanced services by interconnecting (physical and virtual) things based on existing and evolving interoperable information and communication technologies. loT is a networking

Revised Manuscript Received on November 27, 2019.

* Correspondence Author

B. Kalpana, Assistant Professor in the Department of Information Technology, R.M.D Engineering College, Kavaraipettai. Mail: cgkkalpana@gmail.com.

R. Sanjay, currently pursuing a bachelor's degree in the stream of Information Technology at R.M.D Engineering College, Thiruvallur, Tamil Nadu, India. infrastructure for cyber-physical systems (CPS), which are engineered systems that are built from, and depend upon, the seamless integration of computational algorithms and physical components.

The IoT will enable the transformation of sensed or gathered data into intelligent information, thus embedding intelligence into our environment. Besides, the IoT will involve billions of devices that can report their location, identity, and history over wireless connections [2]. Hence there arises a need where the devices have to communicate with each other to share their location and identity. The same way the devices that are forming any network or group have to communication among them to share their information.

There are situations where infrastructure or a central node may fail and there will be a lag in communication between the interconnected devices. The devices have to find another path to reach their destination which can be made possible by making use of multi-hop networks. A multi-hop network is one in which the nodes or devices do not directly transmit their signals to the base station in a single hop, instead, they take multiple hops with the in-between nodes to reach the destination.

The objective of the paper is to propose a mechanism where the devices on the internet of things will make use of intelligent communication mechanisms to communicate with each other, share information or interact with each other, without the increased expenditure of energy and finding the best route to reach the destination. Also, we focus on routing algorithms that will help the packets find their path to the destination node or base station. Also, we throw light on the challenges that will be faced in implementing the proposed communication style.

\section{PROPOSED COMMUNICATION MECHANISM}

Device-to-Device communication has become very popular in recent times. Cellular device to device communication has become an area of interest. Hence there is a need to look into how intelligent communication can be achieved in IoT devices.

There is a primary question of why there is a need for Device-to-Device communication. The answer is that it will enable efficient use of the network resources and it will provide robustness if the central 


\section{A Composite Model for an Intelligent Device-To-Device Communication in the Internet of Things}

infrastructure of the network goes down or becomes unreachable when a device is out of range. A device in a remote location can leverage Device-to-Device communication for a short-range peer-to-peer connectivity, this allows them to share their data. In IoT, communication is expected to be a normal occurrence.

The IoT doesn't have a homogenous network. Due to this heterogeneous nature of IoT intelligence will be required in Device-to-Device communication processes. Intelligence here is the ability to be aware of the environment in which the device is operating. It also defines the environment in which the devices have to collaborate and carry out the desired task. The purpose of a Device-to-Device communication is to initiate some action, synchronize devices in an environment, responding, exchanging information or forwarding data. For this purpose, a device is expected to have the ability to route data to other devices and exchanges the information among them. These devices form a cluster. All the devices in the cluster have to route among themselves and have to communicate with other clusters in the surrounding or independent devices. The purpose of going for a multi-hop group communication

1) Providing service continuity even when the devices are out of range of a base station.

2) Sharing information among all the devices that participate in the group.

3) Off-loading of cellular traffic

4) Provide communication in disaster scenario

5) Automation

Here in the proposed network, the devices that are supposed to form the cluster or the local network are designed to communicate in a network without any fixed infrastructure. The clusters that are formed of the IoT devices are supposed to communicate among themselves by making use of the network with fixed infrastructure. The pattern and advantages are discussed below.

\section{Communication Of Devices In Internet Of Things(IoT) That Form The Cluster}

Link establishment among groups is achieved by paging. Paging is a procedure the network uses to find out a device's location before the actual connection establishment. Secure communication occurs once links are established. The main attribute of this approach is that it facilitates a self-organizing stand-alone device-to-device communication setup without a network infrastructure anytime and anywhere as long as there are devices ready to communicate $[32,33,34]$. The reason for poor adoption is that it is difficult to establish cooperative communication between any two devices unless they belong to a known user. Also, the approach requires the user's manual intervention for a device to be paired with another device.

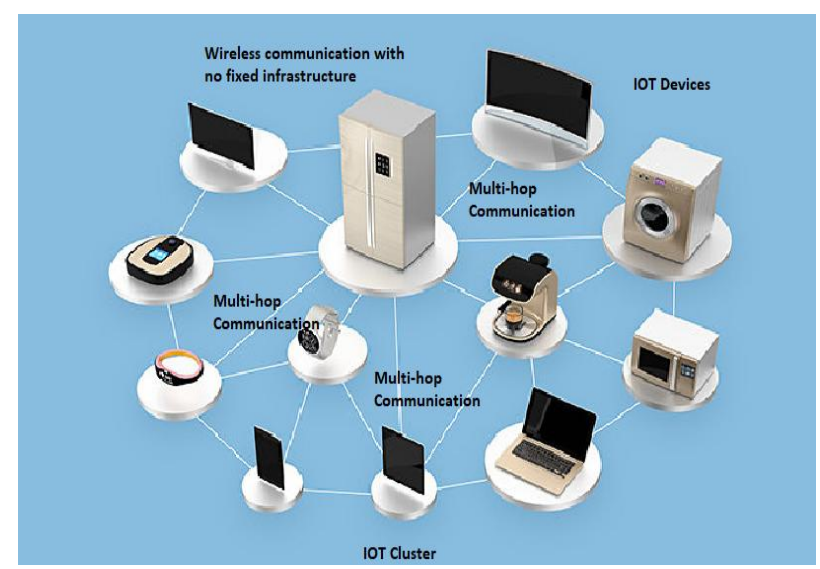

Fig : Proposed group communication among cluster

The WiFi direct mode enables device-to-device connectivity between mobile IoT devices. Other short-range wireless technologies include Bluetooth, ultra-wideband technology, and near field communication are common examples that enable device-to-device communication. Here link discovery and authentication are maintained solely by the devices. Sustained transmission and scanning during the link discovery process consumes a significant amount of energy from the devices. This will not be a major concern in an IoT network since most of the sensors are gonna be within a fixed range and the discovery of devices within the given range will not consume a large amount of energy.

The devices which form the cluster can find the adjacent devices in a single hop if it is the closest device or it can make use of multi-hop fashion to communicate and identify the devices to which the current node(device) has to establish a connection and has to transfer data. This overcomes the need to establish a centralized data repository regarding the location of the devices or to maintain a central connecting device.

\section{Communication Between Clustered Devices In Any Network}

The devices that form a group or cluster will make use of infrastructure-dependent communication for communicating among themselves. Among other procedures, the infrastructure-based network facilitates link discovery and security procedures such as device authentication [20]. Link discovery is a process by which devices find neighboring devices within a range, which is a major concern in a cluster. Moreover, neighboring devices must be available in a device's communication range for connectivity and routing [21].

The link discovery process includes broadcast to other networks to connect with. In a network-dependent communication, the network infrastructure can enable a faster and more energy-efficient link discovery by informing devices about other devices within its range[22, 23]. Hence link discovery is less time consuming and energy-efficient. Hence an infrastructure dependent network can be used for

long-distance 
communication between devices[28].

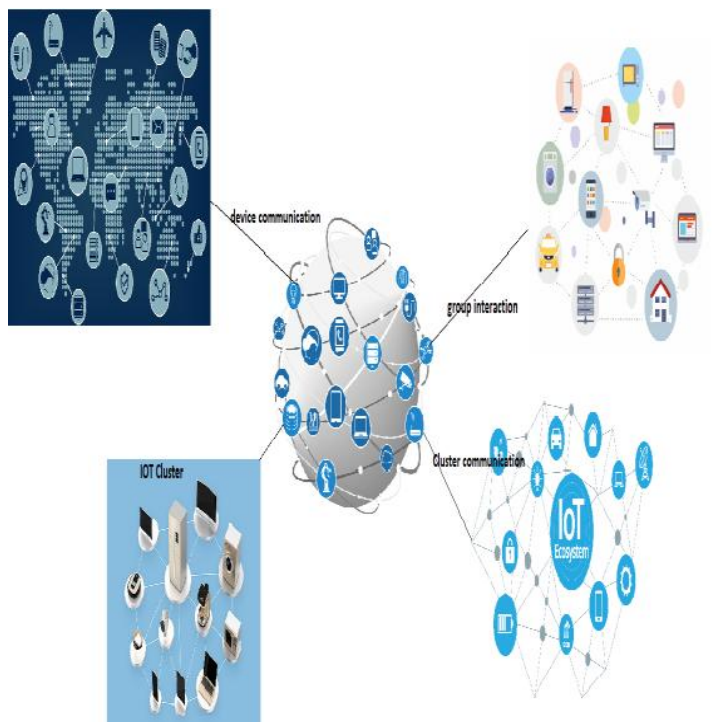

Fig: proposed group interaction architecture

Another limitation is the lack of automated security mechanisms for authenticating devices during the communication link establishment. However, network infrastructure can facilitate automated secure authentication during connection establishment. Link discovery and authentication processes are easier if devices belong to the same network provider.

Limitations in utilizing fixed infrastructure:

i. Devices/ clusters must have cellular network subscriptions [31].

ii. Devices/ clusters must subscribe to the same network [31].

iii. Devices/ clusters in each other's range but not connected to a cellular network infrastructure that can facilitate device-to-device communication will not be able to communicate.

\section{Routing Algorithms / Protocols For intelligent communication in IOT}

IoT devices require the transmission of sensed data between devices or from a set of devices to a central station for analysis or storage. However, the inherent characteristics of a typical device-to-device communication in IoT raise many challenges that traditional routing protocols cannot resolve. Some characteristics of device-to-device communication within the IoT ecosystem include the following.

1. Heterogeneity of devices: Devices differ as a result of their functionality

2. Device coexistence and collaboration: Various devices that can interconnect and communicate anytime in a collaborative manner with any other device will coexist.

3. Diverse networks and networking standards.

4. Device limitations: Devices will be constrained in terms of battery life, memory, and processing power.
5. Self-configuration, self-organization, and autonomy: Most D2D networks will be self-configuring, self-organized, and autonomous. Therefore, devices will handle interference management, translation of different network protocols, and end-to-end communication.

Hence we can say that intelligent IoT communication requires innovative methods to communicate with one another. The solution lies in the heart of the networking process, which is routing[15]. Here we present a classification of routing algorithms/ protocols for the IoT and analyze them concerning the characteristics of the aforementioned device-to-device communication.

\section{Stochastic / Probabilistic Algorithms}

These types of algorithms have particular optimization objectives. They are designed to formulate routing probabilities that optimize a set of network resources such as energy consumption, link metrics, etc. Any selected route is one that maximizes the criteria of interest. Two basic methods used for optimization by these algorithms are real-time optimization and apriori optimization.

In real-time optimization, information about the criteria of interest is revealed as data traffic flows from device to device, thus routes ae dynamically created and on the available information. This method consumes a lot of energy and computational power. For apriori optimization, a solution is determined beforehand and the solution is probabilistically constrained, thus routes that are created mist have probabilities within the set constraints [39].

Most of the routing algorithms in this category have been proposed for wireless networks because of the unstable environment that the wireless medium creates. Therefore, stochastic routing algorithms are better suited to support the unpredictable mobility patterns of devices in device-to-device communication within the IoT.

\section{Bioinspired Algorithms}

This class of algorithms addresses the challenges that are common and significant to large-scale networks, which are characterized by complex and heterogeneous architectures, a dynamic and self-organizing nature, resource constraints, and the absence of centralized control and infrastructure [ 37 38].

The main principles behind the operation of ACO routing algorithms include the following.

1. Foraging: It is a process used by ants to discover the shortest path from their nest to a food source.

2. Stigmergy: It is the process by which ants locally modify discovered paths and react to these modifications, thus creating a form of global coordination among themselves [42].

Features of bio-inspired algorithms that make them 


\section{A Composite Model for an Intelligent Device-To-Device Communication in the Internet of Things}

appealing for the development of routing algorithms for intelligent device-to-device communication include the following.

1. They can handle the heterogeneity and asymmetry in the capabilities and technology of communicating devices within the IoT.

2. They can support self-organizing, self-configuration, and collaboration, which facilitate the setup of autonomous infrastructure-independent device-to-device communication in the IoT environment.

3. Bioinspired algorithms can dynamically adapt to ensure end-to-end communication between devices.

4. Adaptability to changing environmental conditions.

5. Robustness and resiliency.

6. Efficient management of constrained resources.

\section{Hierarchical Algorithms}

Hierarchical algorithms can be tree-based or cluster-based.

Tree-based algorithms: Tree-based algorithms require that devices share the same destination. A tree of multiple hops is dynamically constructed for routing messages and data, which creates a traffic pattern of many to one. This feature limits their general use in the IoT as different communication patterns could exist between devices. For example, communication in a D2D network may be one to one, one to many, many to one, and many to many.

Cluster-based algorithms: Cluster-based algorithms hierarchically classify devices/clusters. Devices have different roles according to their level of the hierarchy. The device with the highest hierarchy in a particular group is the cluster head $(\mathrm{CH})$ or the leader of the group. Typically, the $\mathrm{CH}$ is responsible for communicating the information generated within its cluster to another cluster or the central station/storage device. How to choose a $\mathrm{CH}$ is a major issue with this class of algorithms. Many algorithms have been proposed in the literature on the election of a $\mathrm{CH}$, but these algorithms generate additional delay and complexity, which may not be suitable for many D2D communications in the IoT.

Context-Aware Algorithms: Context is any information that can be used to characterize the situation of an entity (people or objects). In the case of devices, context gives their internal or external status and can be extracted from a device, its environment, or the content of received messages. A system is said to be context-aware if it uses the gathered context to provide relevant services to the user, where relevancy depends on the user's task. Thus, context can be used to provide a routing service to a device when the device's task is to forward data.

A context-aware system or application also can dynamically change or adapt its output or behavior based on the context of the entity it is providing service to. Concerning device-to-device communication, context-aware routing algorithms can obtain relevant information (context) about the situation surrounding devices within a network and select routes through which to forward traffic/data based on the information.

Context-aware algorithms possess the following features:

1. Contextual sensing: the ability $y$ to detect contextual information.

2. contextual adaptation: the ability to execute or modify a service automatically based on the current context.

3 . contextual resource discovery: the ability to locate and exploit relevant resources and services.

4. contextual augmentation: the ability to associate digital data with the user's context.

Although each class of algorithm has its applicability, the classes most suitable for intelligent D2D communication in the IoT are the stochastic, bioinspired, and context-aware algorithms. These algorithms support the characteristics of intelligent D2D communication. They are also able to opportunistically leverage network resources in real-time to allow cognitive and seamless communications.

\section{FUTURE CHALLENGES IN IMPLEMENTING THE PROPOSED MODEL}

\section{Communication Resource Optimization}

Communication resource optimization involves maximizing the use of available network resources. These resources can be classified as global and local resources. Global resources are network resources such as the frequency spectrum, whereas local resources are the resources that are inherent to devices.

The mechanisms for efficient frequency allocation and sharing should be able to do the following.

1. Intelligently share and allocate frequency blocks among network traffic by using criteria, which are determined from signaling or control information received from devices. A criterion may be traffic content or quality of service.

2. Perform adaptive selection and scheduling of frequency blocks for traffic within the network.

\section{Optimized Route Discovery and Management}

Optimized route discovery involves finding the best possible path for end-to-end device-to0-device communication by taking into consideration the networks and device constraints. Route management is the process of finding the best alternative path when a link on an established route fails. to achieve optimized route discovery and management, intelligent protocols are needed. Some factors relevant to the IoT that affect the discovery and maintenance of multihop routes include the following.

i. Mobility

ii. Lack of cooperation between devices $\backslash$

iii. Lack of adequate communication resources

iv. Spectrum switching 


\section{Cooperation Between Devices in the IoT}

Device-to-Device communication in the IoT will typically be multiple hops in nature because devices have to relay traffic for one another, thus performing routing functions. This will necessitate cooperation between devices. Cooperation is a challenge that affects the achievement of optimal routing in device-device communication. Some devices may not collaborate to relay other device's traffic because of reasons such as limited available power, security, and trust. therefore, effective mechanisms for enabling and coordinating cooperation between devices are required. A solution to this challenge is to use human social networking concepts. The authors developed a cooperation strategy based on special trust and social reciprocity for device-to-device communication.

\section{Security}

The provision of security in a complex and heterogeneous IoT ecosystem remains a significant challenge. Considering that device-to-device communication involves interactions between the cyber and the physical world, it is imperative to develop mechanisms that provide reliable security services such as the following.
1. Privacy
2. Authentication
3. Integrity

Security attacks in device-to-device communication include the following.

1. Inference attack

2. Distributed denial-of-service attack

\section{CONCLUSION}

Device-to-Device communication is an integral part of any IoT network. Here we are suggesting a network where the devices in a local network communicate with each other in the infrastructure-less network and these local networks and individual devices communicate with each other by making use of an infrastructure-based network. The reason behind this is that the devices within a cluster are within a short-range and can make use of infrastructure-less networks, where there is no need for setting up a network and base station for these devices. In a very large space, an infrastructure-less network will take a very large amount of time to find the peer network or devices hence a fixed network is required to overcome this overhead. The proposed architecture will provide reliable communication within and between the clusters.

\section{REFERENCES}

[1] J. Gubbi, R. Buyya, S. Marusic, and M. Palaniswami, "Internet of Things (IoT): A vision, architectural elements, and future directions," Future Gener. Comput. Syst., vol. 29, no. 7, pp. 1645-1660, Sep. 2013.

[2] J. Buckley, "From RFID to the Internet of Things pervasive networked systems," Conference Centre Albert Borschette (CCAB), Brussels, Belgium, Mar. 2006. [Online].

[3] D. Evans, "The Internet of things: How the next evolution of the Internet is changing everything," Cisco IBSG, San Francisco, CA, USA, Apr. 2011. [Online].

[4] Y. LeCun, Y. Bengio, G. Hinton, "Deep learning", Nature, vol. 521, pp. 436, 2015.
[5] D. Lake, A. Rayes, and M. Morrow, "The Internet of Things," Internet Protocol J., vol. 15, no. 3, pp. 10-19, Sep. 2012. [Online].

[6] D. Neumann, T. Wiese, W. Utschick, "Learning the MMSE channel estimator", IEEE Trans. Signal Process., vol. 66, no. 11, pp. 2905-2917, Jun. 2018.

[7] Cisco Visual Networking Index: Global Mobile Data Traffic Forecast Update, 2012-2017. Cisco, Feb. 2013. [Online].

[8] H. Ye, L. Liang, G. Y. Li, B.-H. F. Juang, "Deep learning based end-to-end wireless communication systems with conditional GAN as unknown channel", arXiv:1903.02551, 2019.

[9] J. Apcar, "Routing in the Internet of Things/M2M Networks," presented at the Ciscolive365, Melbourne, VIC, Australia, 2013, BRKSPG-1661. [Online].

[10] S. Yu and Y. Peng, "Research of routing protocol in RFID-based Internet of Things," Int. J. Comput. Inf. Technol., vol. 1, no. 2, pp. 94-96, Nov. 2012.

[11] O. Vermesan, M. Harrison, H. Vogt, K. Kalaboukas, M. Tomasella, K.Wouters,K.U.Leuven, S.Gusmeroli,andS.Haller, "Internet of Things strategic research roadmap" in Proc. Eur. Union IoT Cluster Strategic Res. Agenda, pp. 9-51, 2011. [Online].

[12] E. De Poorter, I. Moerman, and P. Demeester, "Enabling direct connectivity between heterogeneous objects in the Internet of Things through a network-service-oriented architecture," EURASIP J. Wireless Commun. Netw., vol. 2011, no. 61, pp. 1-14, Aug. 2011. [Online].

[13] S. Farrell, "Security in the Wild," IEEE Internet Comput., vol. 15, no. 3, pp. 86-91, May/Jun. 2011.

[14] I. Akyildiz, F. Su, W. Sankarasubramaniam, and Y. E. Cayirci, "Wireless sensor networks: A survey," Comput. Netw. (Elsevier) J., vol. 38, no. 4, pp. 393-422, Mar. 2002.

[15] A. Liotta, Why the Internet Needs Cognitive Protocols. New York, NY, USA: IEEE Spectrum, May 2013. [Online].

[16] G. Fodor, E. Dahlman, G. Mildh, S. Parkvall, N. Reider, G. Miklós, and Z. Turányi, "Design aspects of network assisted device-to-device communications,” IEEE Commun. Mag., vol. 50, no. 3, pp. 170-177, Mar. 2012.

[17] K. Doppler and M. Xiao, "Innovative concepts in peer-to-peer and network coding," WINNER+/CELTIC, Munich, Germany, Jan. 2009. [Online]

[18] K. Doppler and M. Xiao, "Device-to-device communication as an underlay to LTE-advanced networks,” IEEE Commun. Mag., vol. 47, no. 12, pp. 42-49, Dec. 2009.

[19] P. Phunchongharn, E. Hossain, and D. In Kim, "Resource allocation for device-to-device communications underlaying LTE-advanced networks," IEEE Wireless Commun., vol. 20, no. 4, pp. 91-100, Aug. 2013.

[20] T. Peng, Q. Lu, H. Wang, S. Xu, and W. Wang, "Interference avoidance mechanisms in the hybrid cellular and device-to-device systems," in Proc. IEEE 20th Int. Symp. Pers., Indoor Mobile Radio Commun., 2009, pp $617-621$.

[21] K. Johnsson, S. Talwar, and N. Himayat, "Mobile device and method for cellular assisted device-to-device communication," WO 2013022471A1, Feb. 14, 2013. [Online]. [22] X. Lin, J. G. Andrews, and A. Ghosh, "A comprehensive framework for device-to-device communications in cellular networks," to be published. [Online].

[23] G. Fodor, M. Belleschi, D. D. Penda, A. Pradini, M. Johansson, and A Abrardo, "Benchmarking Practical RRM Algorithms for D2D Communications in LTE Advanced," in Proc. CoRR, 2013.

[24] P. Janis, V. Koivunen, C. Ribeiro, J. Korhonen, K. Doppler, and K. Hugl, "Interference-aware resource allocation for device-to-device radio underlaying cellular networks," in Proc. IEEE 71st VTC, 2009, pp. 1-5.

[25] G. Fodor and N. Redier, "A distributed power control and mode selection scheme for device-to-device communications," in Proc. IEEE Globecom, Houston, TX, USA, 2011, pp. 1-6.

[26] M. Belleschi, G. Fodor, and A. Abrardo, "Performance analysis of a distributed resource allocation scheme for D2D communications," in Proc. IEEE Globecom, Houston, TX, USA, 2011, pp. 358-362.

[27] B. Wang, L. Chen, X. Chen, X. Zhang, and D. Yang, "Resource allocation optimization for device-to-device communication underlaying cellular networks," in Proc. IEEE 73rd VTC, 2011, pp. 1-6.

[28] A. Osseiran, "Advances in device-to-device communications and network coding for IMT-advanced," in Proc. ICT-Mobile Summit, 2009, pp. 1-8.

[29] T. Koskela, V. Van Phan, G. Charbit, and S. Hakola, "Method and apparatus for providing communication offloading to unlicensed bands," Patent No.20110287794,Nov. 24,2011.[Online].

[30] Proximity communication, 2010. [Online].

[31] G. Fodor and M. Kazmi, "User equipment and a radio network node, and methods therein for device-to-device communication," Patent No. WO 2013055271 A1, Apr. 18, 2013. [Online].

[32] R. Laroia, "Future of wireless? The proximate Internet," presented at the COMSNETS, Bangalore, India, Jan. 5-9, 2010. 


\section{A Composite Model for an Intelligent Device-To-Device Communication in the Internet of Things}

[33] J. Perez-Romero, O. Salient, R. Agusti, and L. Giupponi, "A novel ondemand cognitive pilot channel enabling dynamic spectrum allocation," in Proc. 2nd Int. Symp. New Frontiers Dyn. Spectrum Access Netw., Apr. 2007, pp. 46-54.

[34] Y. Chia-Hao, K. Doppler, C. B. Ribeiro, and O. Tirkkonen, "Resource sharing optimization for device-to-device communication underlaying cellular networks," IEEE Trans. Wireless Commun., vol. 10, no. 8, pp. 2752-2763, Aug. 2011.

[35] K. Machado, D. Rosário, E. Cerqueira, A. Loureiro, A. Neto, and J. de Souza, "A routing protocol based on energy and link quality for Internet of Things applications," Sensors, vol. 13, no. 2, pp. 1942-1964, 2013.

[36] C. Nussbaum, Biologically Inspired Routing-The Future of Networks, Aug. 22, 2013. [Online].

[37] F. Dressler and O.Akan, "A surveyonbio-inspired networking," Comput Netw., Int. J. Comput. Telecommun. Netw., vol. 54, no. 6, pp. 881-900, Apr. 2010.

[38] M.FarooqandG.DiCaro,"Routingprotocolsfornetgenerationnetworks inspired by collective behaviours of insect societies: An overview," in Swarm Intelligence: Introduction and Applications. Berlin, Germany: Springer-Verlag, 2008, ser. Springer Natural Computing.

[39] D. Papadimitriou, "Stochastic Routing," in Routing Area Meeting, IETF 82, Taipei, Taiwan, Nov. 2011. [Online].

[40] A. Liotta and G. Exarchakos, "A peek into the future," in Networks for Pervasive Services. New York, NY, USA: Springer-Verlag, 2011, pp. $145-155$.

[41] E. Bonabeu, M. Dorigo, and G. Theraulaz, Swarm Intelligence: From Natural to Artificial Systems. London, U.K.: Oxford Univ. Press, 1999.

[42] G. Di Caro, F. Ducatelle, and L. M. Gambardella, "AntHocNet: An adaptivenature-inspiredalgorithmforroutinginmobileadhocnetworks,"Eu r Trans. Telecommun., Special Issue Self-org. Mobile Netw., vol. 16, no. 5, pp. 443-455, Sep./Oct. 2005.

[43] B. Atakan and O. B. Akan, "Biologically-inspired spectrum sharing in cognitive radio networks," in Proc. IEEE Wireless Commun. Netw. Conf., Kowloon, Hong Kong, 2007, pp. 43-48.

[44] W. Vogels, R. van Renesse, and K. Briman, "The power of epidemics: Robust communication for large-scale distributed systems," ACMSIGCOMM Computer Communication, vol. 33, no. 1, pp. 131-135, Jan. 2003.

[45] T. Tsuchiya and T. Kikuno, "An adaptive mechanism for epidemic communication," in Proc. 1st Int. Workshop Biologically Inspired Approaches Adv. Inf. Technol., Lausanne, Switzerland, 2004, pp. 306-316.

[46] I. Carreras, D. Miorandi, G. S. Canright, and K. Engo-Monsen, "Understanding the spread of epidemics in highly mobile networks," in Proc. 1st IEEE/ACM Int. Conf. Bio-Inspired Models Netw., Inf. Comput. Syst., Cavalese, Italy, 2006, pp. 1-8.

[47] G. Abowd, A. Dey, P. Brown, N. Davies, M. Smith, and P. Steggles, "Towards a better understanding of context and context-awareness," in Proc. Handheld Ubiquitous Comput., 1999, pp. 304-307.

[48] V. Carlvaho, "Including context in a routing algorithm for the Internet of Things," Ph.D. dissertation, Dept. de Engenharia Informatica, Universidade Nova de Lisboa, Lisbon, Portugal, 2010. [49] M. Musolesi and C. Mascolo, "Evaluating context information predictability for autonomic communication," in Proc. WOWMOM, Buffalo-Niagara Falls, NY, USA, 2006, pp. 495-499.

[50] B. Wenning, A. Timm-Giel, and C. Gorg, "A generic framework for context-aware routing and its implementation in wireless sensor networks," in Proc. Technologien und Anwendungen, Vortrage der 14. ITG-Fachtagung, Osnabruck, Germany, 2009, pp. 53-58.

[51] K.-C. Chenand S.-Y. Lien, "Machine-to-machine communications: Technologies and challenges," Adhoc Networks, 2013. [Online]. Available: http://dx.doi.org/10.1016/j.adhoc.2013.03.007

[52] X. Chen, B. Proulx, X. Gong, and J. Zhang, "Social trust and socia reciprocity based cooperative D2D communications," in Proc ACM Int. Symp. MOBIHOC, Bangalore, India, 2013, pp. 187-196.

[53] S. Zeadally, A. Pathan, C. Alcaraz, and M. Badra, "Towards privacy protection in smart grid," Wireless Pers. Commun., vol. 73, no. 1, pp. 23-50, Nov. 2013

[54] 3GPP TS 11.113rd Generation Partnership Project; Technical Specification Group Terminals Specification of the Subscriber Identity Module - Mobile Equipment (SIM- ME) interface.

[55] O. ElGarhy, L. Reggiani, "Increasing efficiency of resource allocation for D2D communication in NB-IoT context", Procedia computer science, vol. 130, pp. 1084-1089, 2018.

[56] D. Feng, L. Lu, Y. Yuan-Wu, G. Y. Li, G. Feng, S. Li, "Device-to-device communications underlying cellular networks", IEEE Transactions on Communications, vol. 61, no. 8, pp. 3541-3551, 2013.

[57] R. Ratasuk, B. Vejlgaard, N. Mangalvedhe, A. Ghosh, "NB-IoT system for M2M communication", Wireless Communications and Networking Conference (WCNC) 2016 IEEE, pp. 1-5, 2016.

\section{AUTHORS PROFILE}

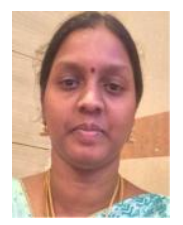

Dr. Kalpana B is an Assistant Professor in the Department of Information Technology, R.M.D Engineering College, Kavaraipettai. She did her M.E Embedded Systems Technologies from Anna University. She did her Ph.D in Information and Communication Technology from Anna university, Chennai. Dr.Kalpana's research areas of interest include adaptive video streaming and IOT for Automation. She has published papers in peer reviewed international journals of repute. She has applied for Research Proposal in Science and Engineering Research Board and is accepted for evaluation.

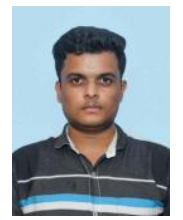

Sanjay $\mathbf{R}$ currently pursuing a bachelor's degree in the stream of Information Technology at R.M.D Engineering College, Thiruvallur, Tamil Nadu, India.He is interested in the fields of Internet of Things, Machine Learning and Wireless Communication. He has presented several papers on these topics at several international conferences. Sanjay has done several projects in the field of Internet of Things and his research interests include device-to-device communication and security in Internet of Things 